\title{
The Evaluation of the Hemocompatibility of Polymer Membrane Materials for Blood Oxygenation
}

\author{
A. Yu. Alentiev ${ }^{a}$ *, Yu. G. Bogdanova ${ }^{b}$, V. D. Dolzhikova ${ }^{b}$, N. A. Belov ${ }^{a}$, R. Yu. Nikiforov ${ }^{a}$, \\ D. A. Alentiev ${ }^{a}$, G. O. Karpov ${ }^{a}$, M. V. Bermeshev ${ }^{a}$, N. V. Borovkova ${ }^{c}$, A. K. Evseev ${ }^{c}$, \\ M. S. Makarov ${ }^{c}$, I. V. Goroncharovskaya ${ }^{c}$, M. V. Storozheva ${ }^{c}$, and S. V. Zhuravel ${ }^{c}$ \\ ${ }^{a}$ Topchiev Institute of Petrochemical Synthesis, Russian Academy of Sciences, Moscow, 119991 Russia \\ ${ }^{b}$ Department of Chemistry, Moscow State University, Moscow, 119991 Russia \\ ${ }^{c}$ Sklifosovsky Research Institute of Emergency Medicine, Moscow, 129090 Russia \\ *e-mail: alentiev@ips.ac.ru
}

Received July 18, 2020; revised July 30, 2020; accepted August 10, 2020

\begin{abstract}
A comprehensive interdisciplinary study is performed for 12 polymers promising for the fabrication of membranes for extracorporeal membrane oxygenation based on them by methods of gas permeability, wetting, piezoelectric microweighing, and direct biomedical methods for determining hemocompatibility of whole blood from healthy donors. It is found that trimethylsilyl-substituted polytricyclononene, polyhexafluoropropylene, and semicrystalline polyphenylene oxide are the best polymer materials for the diffusion membranes of oxygenators. It is shown that traditional approaches that associate the surface properties of polymers (water wettability, plasma protein adsorption, energy characteristics of surfaces) with their hemocompatibility do not provide precise correlations with the biomedical methods based on the analysis of the changes in the shape of blood cells as a result of adhesion on the surface of the polymer. The complexity of the mechanism of interaction of the surface of polymers with blood also does not allow for clear structureproperty correlations traditional for membrane gas separation. The directions of further research in this area are defined.
\end{abstract}

Keywords: polymers, membranes, gas permeability, hemocompatibility, wetting, interphase energy, adsorption, extracorporeal membrane oxygenation

DOI: $10.1134 / \mathrm{S} 2517751620060025$

\section{INTRODUCTION}

One of the most effective methods for the treatment of acute respiratory failure is extracorporeal membrane oxygenation (ECMO) or "iron lung" based on providing a systemic bloodstream using a centrifuge pump and extracorporeal oxygen saturation of blood and elimination of carbon dioxide dissolved in blood using membrane gas exchange [1,2]. This technology is used for life support when conducting longterm open-heart surgeries; when performing heart, lung, liver, and cardiopulmonary complex transplant surgeries as well as in case of traumas associated with massive blood loss [1]. Until recently, a less common field of application of this technology has been the application for the treatment of severe pneumonias of different etiologies [3]. During the period of evolution of the COVID-19 pandemic, ECMO technology became a prime life-saving method in severe cases [4]. Thus, according to the data of the Euro ECMOCOVID Survey observational study [5], 1336 cases of the use of ECMO technology for saving patients with an extreme infection caused by the SARS-CoV-2 virus have been recorded over 4 months of the pandemic from March to July in the European countries including 46 in Russia [6]. At the same time, 711 patients were successfully weaned from ECMO with improvement in their condition.

The key element of modern ECMO technology is an oxygenator, a hollow-fiber membrane contactor, in which a polymer membrane in the form of a hollow fiber separates gas (oxygen or air) and liquid (blood) phases [1, 2]. Generally, a gas flow is supplied onto the internal surface of the fiber, and the external surface of the membrane is washed with blood. The efficiency and duration of operation of an ECMO oxygenator directly depend on the properties of the material such as hemocompatibility (absence of structural and functional abnormalities of the cells of blood and its plasma composition upon contact with the material) and thromboresistance (the prevention of spontaneous coagulation of blood and formation of blood clots on the surface of the material). So-called diffusion membranes (generally, composite), in which a thin nonporous (diffusion) layer of a hemocompatible polymer is applied onto the surface of porous hollow fibers are used for the long-term (up to several days 
and even weeks) applications of ECMO technology [2]. Oxygen that passed through the polymer membrane dissolves in blood in the molecular form by the dissolution-diffusion mechanism, while carbon dioxide dissolved in blood desorbs through the membrane to the gas phase. The efficiency of oxygen transport to blood and transfer of carbon dioxide in the diffusion mode depends on the surface area of the membrane and permeability of the surface diffusion layer of the polymer, e.g., its thickness and permeability of the polymer material $[1,2]$. In state-of-the-art oxygenators, polypropylene or polymethylpentene (PMP) is generally used as the porous hollow fiber [1, 2], while various modifications of cross-linked polydimethylsiloxane (PDMS) with grafted biocompatible thromboresistant coatings containing heparin or phosphoryl choline are used as the material of the diffusion coating $[1,2]$, which makes it possible to achieve the duration of operation of some oxygenators of up to 30 days upon performing ECMO [2]. Despite all the advantages of PDMS (a highly permeable highly elastic polymer allowed for medical use in contact with blood), a series of serious disadvantages cannot be ruled out as well.

(1) For PDMS, the following upper boundary of permeability is specified in the published sources [7]: oxygen permeability coefficient $P\left(\mathrm{O}_{2}\right)=600$ Barrer (1 Barrer $=10^{-10} \mathrm{~cm}^{3}(\mathrm{STP}) \mathrm{cm} \mathrm{cm}^{-2} \mathrm{~s}^{-1} \mathrm{~cm} \mathrm{Hg}^{-1}$ ), although this value may be substantially lower in the real material of the coating due to the different densities of the crosslinks. Here, other materials currently applied for continuous layers have substantially lower oxygen and carbon dioxide permeability coefficients [2].

(2) It is generally impossible to create the continuous layer for the membranes made of cross-linked PDMS or copolymers and block copolymers of PDMS with a thickness of less than 3-5 $\mu \mathrm{m}$ like in gas separation membranes [8], hence, the gas flux through the membrane is limited by the permeability of the material (which decreases with the increase in the density of the crosslinks and presence of fillers) and thickness of the diffusion layer.

(3) An increased blood protein sorption on the surface is observed for PDMS materials, which leads to blood clot formation at prolonged operation times [9, 10]. To prevent the absorption of fibrinogen and blood cells, biocompatible thromboresistant coatings are applied onto the surface of the diffusion layer [1,2].

It is often mentioned in the published sources that semicrystalline PMP is often the material for the continuous diffusion layer of ECMO membranes; however, it is substantially inferior to PDMS with respect to permeability $\left(P\left(\mathrm{O}_{2}\right)=27\right.$ Barrer [11]). Nevertheless, many polymer materials are known by now which possess gas permeabilities that are substantially higher when compared to both PMP and PDMS [12]. Entire classes of highly permeable glass-like amorphous polymers such as substituted polyacetylenes [13], polynorbornenes and polytricyclononenes [14], perfluorinated amorphous polymers [15], polymers with internal microporosity (PIM polymers) $[16,17]$, and polyimides and polyamides [17] have been studied among these materials. However, almost no hemocompatibility studies have been performed among such polymers promising for the creation of continuous diffusion composite membranes, although it has been shown for perfluorinated amorphous polymers [18] that they possess a favorable combination of gas permeability and hemocompatibility. Therefore, the search for new highly permeable and hemocompatible polymers that can compete with PMP and PDMS and even perfluorinated amorphous polymers with respect to their characteristics (including cost) [18] is very relevant.

Apparently, such a search cannot be solely based on the medical and biological experimental hemocompatibility indices of polymer materials (structural integrity, morphological and functional stability of the cell structures of blood, adhesion and aggregation of platelets on the surface of the polymer [19-22]). It is correct to compare these indices only within a specific sample or upon accumulating a large amount of statistical data in connection with the fluctuations of individual blood values as a result of natural physiological instability.

Since the contact of the material of the oxygenator with blood is particularly implemented on the surface of the membrane, the determination of the characteristics of the surface of polymer films and selection of the criterial physicochemical selection parameters for them can substantially optimize the choice of polymers promising for application in contact with blood for medical and biological testing. Such an optimization will make it possible to exclude from consideration the objects for which direct experiments on hemocompatibility are a priori unreasonable.

The problem of interrelation of the surface properties of polymer materials and their hemocompatibility has been discussed in scientific publications for over 50 years; however, no universal approach has been developed until now, which would have allowed unambiguously predicting hemocompatibility based on the physicochemical tests of the material/blood interface.

The characteristics of the surface can affect the hemocompatibility indices such as adhesion of blood cells and adsorption of plasma proteins. These indices are interrelated. The adsorption of fibrinogen, being one of the largest plasma proteins readily changes the conformation as a result of adsorption, should be minimum for a thromboresistant material. This change is one of the factors triggering the cascade mechanism of blood clot formation [23]. The threshold value of adsorption of fibrinogen is also known, which leads to the initiation of this mechanism, it is $0.02 \mu \mathrm{g} / \mathrm{cm}^{2}$ [24]. Nevertheless, the correlation between the adsorption 


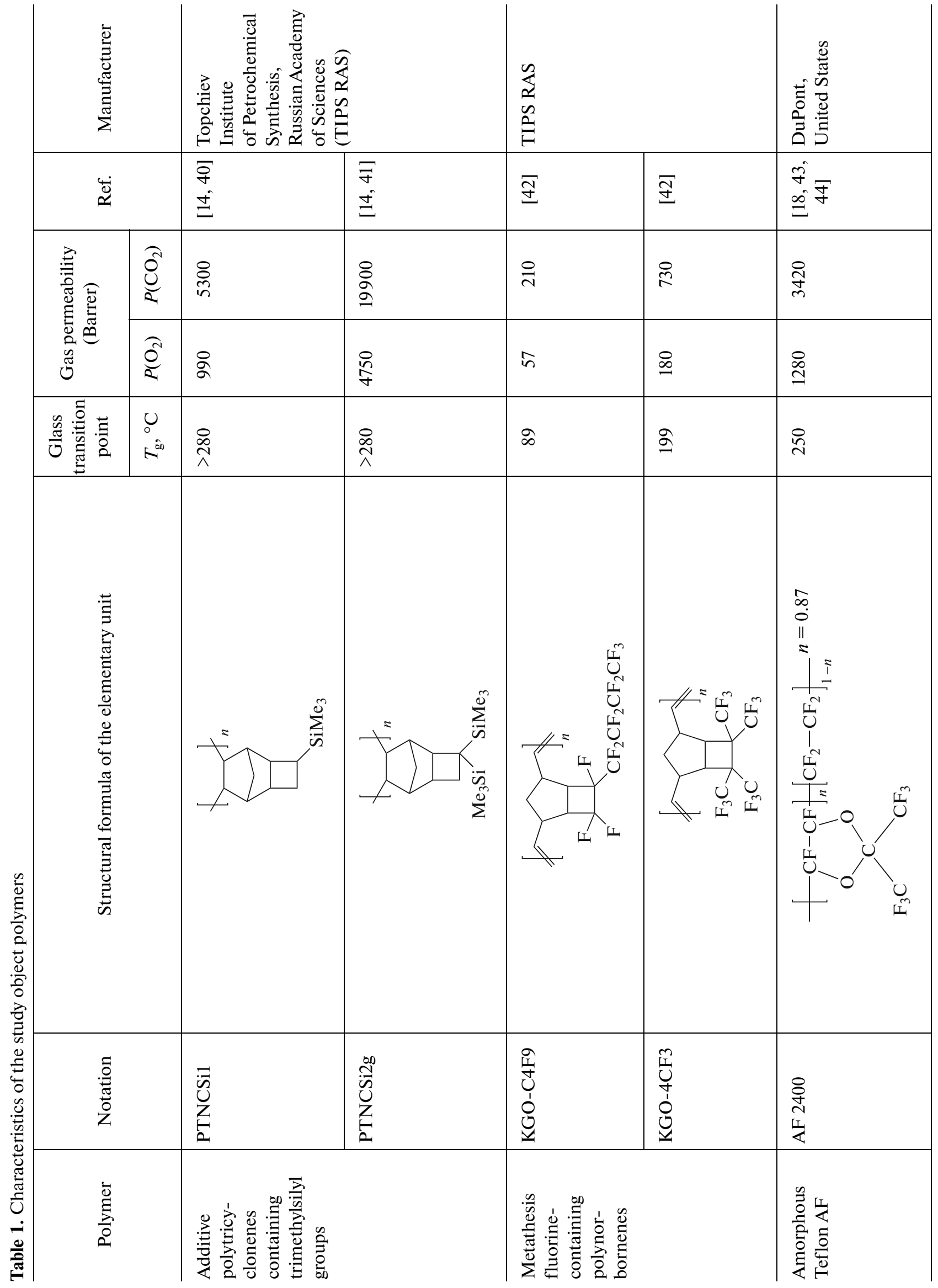




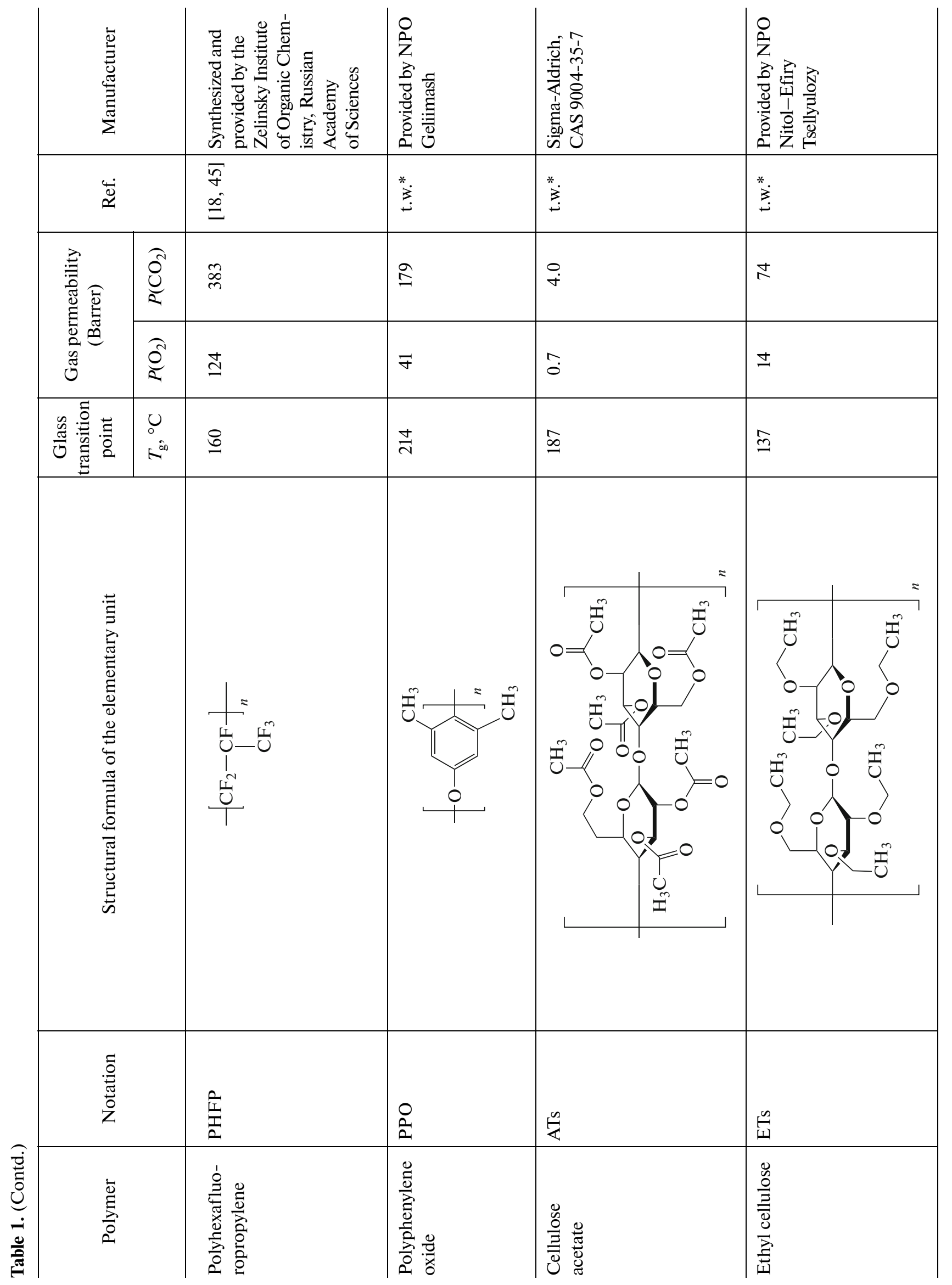


ALENTIEV et al.

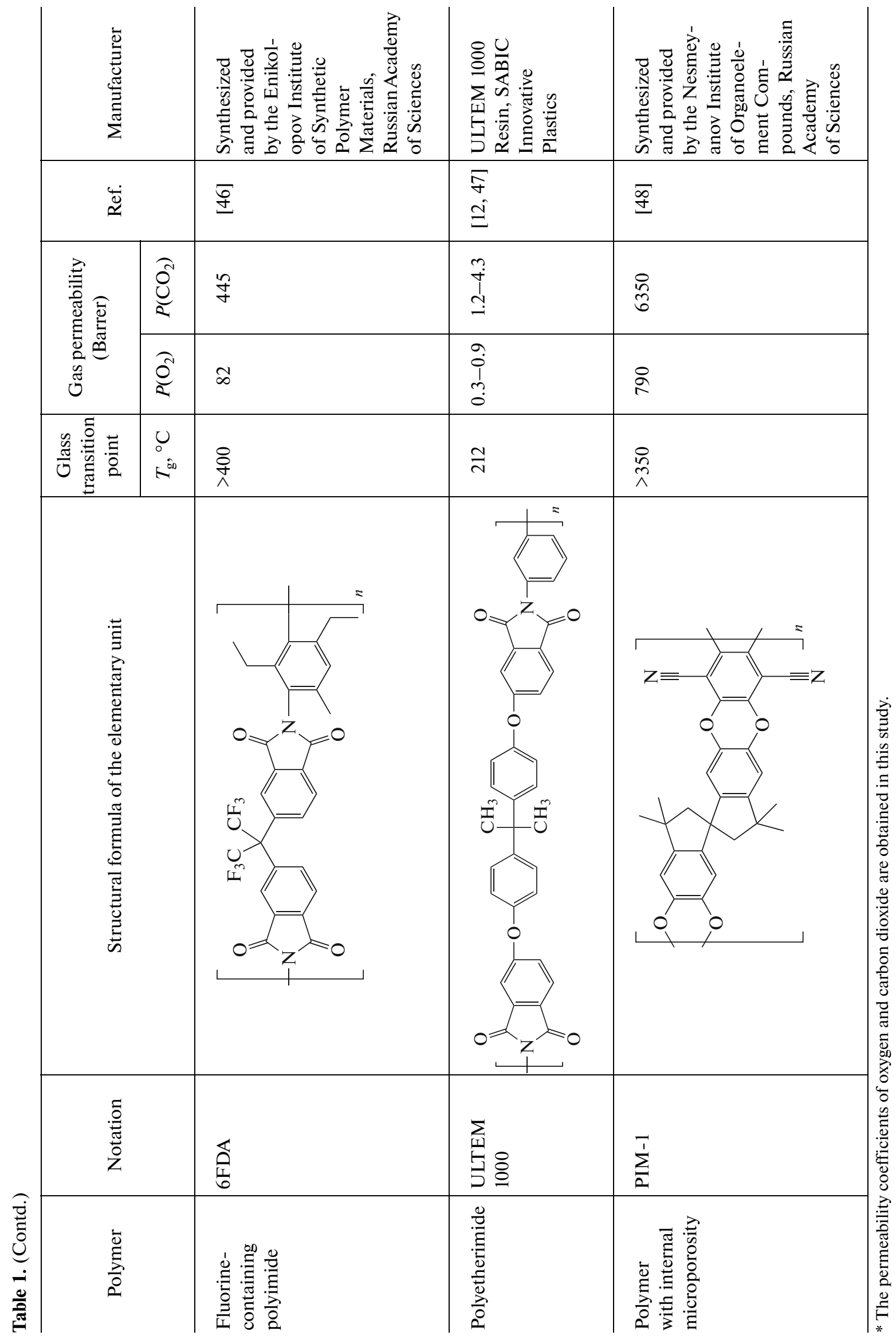

MEMBRANES AND MEMBRANE TECHNOLOGIES Vol. 2 No. 62020 
of fibrinogen and capacity of platelets for adhesion on the modified polymer surface is ambiguous because the adhesion of platelets is affected not only by the amount of adsorbed fibrinogen but also by its molecular activity [25]. On the opposite, the adsorption layers of albumin demonstrate protector properties in relation to the adhesion of platelets provided that the protein retains the native conformation in the adsorption layer. A change in the conformation of albumin as a result of adsorption leads to the intensification of the adhesion of leucocytes and macrophages and promotes the development of inflammatory processes in the point of contact of the polymer material with blood and degradation of the polymer [26, 27]. It should be noted that the mechanical stability of the polymer material in the biological environment is needed for providing its functions [26, 28].

All the specified characteristics are to a great extent determined by the values of the specific surface free energy of the polymer films and interphase energy of the polymer/blood surface, which can be calculated based on the experimental values of the contact angles of liquids on the surface of polymers under various conditions [29-31]. Blood is a complex system, because of which the evaluation of the hemocompatibility of polymer materials in the physicochemical context is performed based on the experiments in model systems. The simplest system modeling blood is water or a solution of a protein (serum albumin, fibrinogen, gamma globulin) or a mixture of proteins. It is worth noting that it should be taken into account upon analyzing the results of the experiments on wetting of the surfaces of polymers by multicomponent liquids that the equations of the molecular theory of wetting have been obtained for the case of pure liquids. The fact that stationary equilibrium conditions (do not take into account the different blood flow velocities in the circulatory system) are often used and the effects associated with the change in curvature of the interface or temperature are not taken into consideration when constructing phenomenological models is also not unimportant $[29,30]$.

Most scientific studies are limited by the analysis of the interrelation between the wettability of the surface of polymer materials by water (hydrophilicity/hydrophobicity) and their hemocompatibility, the index of which is considered to be the adhesion of cells and/or adsorption of plasma proteins to the surface of the polymer. No unambiguous correlation between the values of the contact angles of water drops (maximum contact angles) and characteristics of the surface, which define hemocompatibility, have been found $[29,32,33]$. The situation is aggravated by the possible lability of the surface groups of the polymer upon contact with blood, which leads to an increase in the hydrophilicity of the polymer [29]. There is still no consensus about how the wettability of the surface of a polymer by water is related to the hemocompatibility of a polymer material [34-36]. This is determined by the very limited amount of systematic interdisciplinary studies in this field among which the following directions can be distinguished:

(1) The comparison of the values of $\gamma_{S V}^{d}$ and $\gamma_{S V}^{p}$ with the adhesion of blood cells (most often, platelets) and traumability of erythrocytes [36, 37].

(2) The comparative analysis of the polymer/water interphase energy and adsorption of plasma proteins on the surface a polymer [29, 30, 37].

(3) The analysis of the work of adhesion of blood and its components to the surface of the polymer [38, 39].

When solving the problem of selection of the polymer for ECMO membranes, it is important to take into account not only the surface and medical and biological characteristics of polymer films but also the parameters of their gas permeability.

A comprehensive study of a series of amorphous glass-like highly permeable polymers promising for the creation of ECMO membranes has been performed in this study. These polymers belong to the above-described classes: additive silicon-containing polynorbornenes, metathesis fluorine-containing polynorbornenes, perfluorinated amorphous polymers, semicrystalline polymers (polyphenylene oxide, cellulose esters), polyimides, and a polymer with internal microporosity (PIM-1).

\section{EXPERIMENTAL}

The structural formulae of the elementary units and physicochemical characteristics of the studied polymers are presented in Table 1.

The polymer films for the measurement of gas permeability and biomedical studies were prepared by casting onto a cellophane substrate from solutions in organic solvents (1-5 wt \%). Reagent grade toluene was used for polytricyclononenes, reagent grade perfluorotoluene was used for fluorine-containing polynorbornenes and perfluorinated polymers, reagent grade acetone was used for cellulose acetate, and reagent grade chloroform was used for the rest of the polymers. For the studies by wetting and piezoelectric microweighing, the films were applied from the corresponding solutions onto solid substrates, an aluminum plate and sensors of piezoquartz balances.

The gas permeability of oxygen and carbon dioxide for the polymer films with a thickness of $\sim 40 \mu \mathrm{m}$ were determined by a barometric procedure on an MKS Barotron unit with the LabView software. Permeability coefficients $P$ were calculated by the temporal dependence of the change in the pressure in a calibrated receiving volume after the mass transport process reached the stationary mode. The error of the measurement of $P$ was 5\%. The experiments were carried out at $35^{\circ} \mathrm{C}$, the pressure over the membrane of about $1 \mathrm{~atm}$, and the pressure after the membrane of about $10^{-3} \mathrm{mmHg}$. 
The energy characteristics of the surfaces of the polymer films, specific surface free energy $\gamma_{\mathrm{Sv}}$ and its dispersion $\gamma_{\mathrm{SV}}^{\mathrm{d}}$ and polar $\gamma_{\mathrm{SV}}^{\mathrm{p}}$ components, were calculated using the experimental values of the maximum contact angles ${ }^{1}$ of water and diiodomethane in accordance with the double-liquid Owens-Wendt-Kaelble approach within the molecular theory of wetting [31]. Interphase energy $\gamma_{\mathrm{s}(\mathrm{W}) \mathrm{w}}$ of the polymer/water boundary (the blood plasma model) was determined by the procedure of E. Ruckenstein [29] with the use of the contact angles of water and octane measured under the conditions of inflow ${ }^{2}$ and selective wetting ${ }^{3}$ after 24-h contact of the polymer films with the test liquids (water, octane). This original procedure was developed for the consideration of the possible change in the surface layer of a polymer in the case of a longterm contact with water (e.g., swelling, reorientation of labile surface groups). The contact angles were measured on an MG-1 horizontal microscope with a goniometric accessory at $20^{\circ} \mathrm{C}$ with the accuracy of $\pm 1^{\circ}$. When determining the average values of the contact angles, the data of five to six measurements were taken into account, the error of which was no more than the accuracy of the measurement of the contact angles, i.e., the data of wetting experiments were distinguished by good reproducibility. Taking this into account, the accuracy of determination of the energy characteristics of the surfaces was no less than $\pm 1^{\circ} \mathrm{mJ} / \mathrm{m}^{2}$. Hereinafter, all the values of $\theta$ and $\gamma$ are presented in the tables taking into account the accuracy of their determination.

The adsorption of the components from the blood plasma of the donors was determined by a direct gravimetric method of piezoelectric weighing (PW) [49] using AT-cut quartz resonators with silver electrodes and the intrinsic frequency of vibrations $f \mathrm{o}=5 \mathrm{MHz}$ as the sensor of the microbalances. The sensors of the microbalances with the polymer films chosen for this experiment applied onto them were exposed at $20^{\circ} \mathrm{C}$ for $30 \mathrm{~min}$ in aliquots of the blood plasma of the donors and then dried. The adsorption of the components of plasma $\Gamma, \mathrm{g} / \mathrm{cm}^{2}$, was calculated by the equation

$$
\Gamma=-1.76 \times 10^{-8} \Delta f,
$$

where $\Delta f$ is the change in the frequency of the sensor of the microbalances.

The determination of free hemoglobin upon contact of the polymer films with the erythrocyte suspension from healthy volunteer donors prepared on a TsFD/S.A.G.M. hemopreservative for $1 \mathrm{~h}$ at $37^{\circ} \mathrm{C}$ was performed by spectrophotometry by a method [50] on

\footnotetext{
${ }^{1}$ The contact angle of a liquid drop on the surface of a polymer film in an air atmosphere.

2 The contact angle of an air bubble on the surface of a polymer film immersed into a liquid.

${ }^{3}$ The contact angle of a liquid on the surface of a polymer film immersed into a different liquid.
}

a DU800 spectrophotometer (Beckman Coulter, United States).

The biomedical hemocompatibility study of the polymer materials was performed in whole blood from healthy volunteer donors obtained using a Vacutainer Hemogard vacuum blood collection system (BD, Great Britain) with a K2EDTA anticoagulant. The samples of the polymers were exposed with the biological medium at $37^{\circ} \mathrm{C}$ for $1 \mathrm{~h}$ after which the microscopic examination of the following indices was performed:

-The concentration of the main morphological types of erythrocytes, $\%$.

-The concentration of free hemoglobin, $\mathrm{g} / \mathrm{L}$.

-The concentration of biologically native platelets, $\%$.

-The morphofunctional status of platelets, scores.

-The formation of platelet aggregates in the cell suspension and on the surface of the polymers (the size of the aggregates, presence of granules in the cells forming the aggregate, distribution of the aggregates on the surface of the polymer).

-The structural integrity of the leucocytes, $\%$ of damaged leucocytes of the entire population.

- The adhesion of leucocytes on the surface of the polymers, ths. cells $/ \mathrm{cm}^{2}$.

The evaluation of the morphology of the cell structures of blood and their aggregation was performed using a Nikon Eclipse 80i confocal microscope (Nikon, Japan) combined with a Nikon Intenslight C-HGFi fluorescent lamp (Nikon, Japan). The vital staining for the morphofunctional analysis of the cells was performed by the procedures set out in [51, 52]. The vital staining makes it possible to evaluate the structure and functions of the cells without disturbing their viability and makes it possible to detect cells on the surface of optically dense substrates.

\section{RESULTS AND DISCUSSION}

The methodology of the study consisted in the following. The studies of the characteristics of the surfaces of the films and biomedical hemocompatibility tests were performed in parallel for the selected series of polymers with the measured permeability coefficients of oxygen and carbon dioxide. Based on the analysis of a set of results of biomedical and physicochemical studies, the most promising polymers for the creation of blood oxygenation systems were chosen from the range of study objects.

\section{The Surface Properties of Polymer Films}

The experimental values of the contact angles of liquids on the surfaces of polymer films determined under various conditions are presented in Table 2.

The studied films are characterized by different water wettabilities. The maximum contact angles on 
Table 2. Average values of the contact angles in the polymer/liquid systems measured under various conditions: $\theta_{\mathrm{a}}-$ inflow (the angle of a liquid drop on a solid surface in an air atmosphere); $\theta_{\mathrm{r}}$ and $\theta_{\mathrm{V}}$-outflow (the angle measured upon bringing an air bubble to a surface immersed into a liquid); $\theta_{\mathrm{r}}$-the counting of the angle towards water; $\theta_{\mathrm{V}}-$ the counting of the angle towards air; and $\theta_{\mathrm{O}}$-selective wetting (the angle of a water drop on a surface immersed into octane counted towards octane)

\begin{tabular}{l|c|c|c|c|c|c}
\hline \multirow{2}{*}{ Polymer } & \multicolumn{2}{|c|}{ Maximum contact angles, deg } & \multirow{2}{*}{$\theta_{\mathrm{r}}, \mathrm{deg}$} & \multicolumn{2}{c}{$\Delta \theta, \mathrm{deg}$} & \multicolumn{2}{c}{ Angles by procedure [29], deg } \\
\cline { 2 - 3 } & $\theta_{\mathrm{a}}\left(\mathrm{H}_{2} \mathrm{O}\right)$ & $\theta_{\mathrm{a}}\left(\mathrm{CH}_{2} \mathrm{I}_{2}\right)$ & & & $\theta_{\mathrm{V}}$ & $\theta_{\mathrm{O}}$ \\
\hline PTCNSi1 & 102 & 46 & 82 & 20 & 141 & 93 \\
PTCNSi2g & 101 & 47 & 82 & 19 & 147 & 96 \\
KGOC4F9 & 97 & 73 & 59 & 38 & 153 & 138 \\
KGO4CF3 & 92 & 60 & 61 & 31 & 149 & 141 \\
AF 2400 & 114 & 93 & 112 & 2 & 63 & 0 \\
PHFP & 119 & 88 & 116 & 3 & 71 & 0 \\
PPO & 86 & 29 & 75 & 11 & 140 & 98 \\
ATs & 66 & 53 & 49 & 17 & 123 & 108 \\
ETs & 81 & 44 & 58 & 23 & 124 & 104 \\
6FDA & 74 & 29 & 74 & 0 & 139 & 131 \\
ULTEM 1000 & 86 & 42 & 78 & 8 & 126 & 103 \\
PIM-1 & 84 & 34 & 84 & 0 & 153 & 127 \\
\hline
\end{tabular}

the surface of PHFP and AF 2400 fluorinated polymers are comparable between each other and with the values of $\theta_{\mathrm{a}}\left(\mathrm{H}_{2} \mathrm{O}\right)=118^{\circ}-120^{\circ}$ on the surface of polytetrafluoroethylene plates [53]. The surfaces of KGOC4F9 and KGO4CF3 fluorine-containing metathesis polynorbornenes are substantially less hydrophobic, and 6FDA fluorinated polyimide is wetted by water, which may be associated with the lower density of the fluorine-containing functional groups in the surface layer of its film. The contact angles of water drops on the surfaces of additive polynorbornenes containing trimethylsilyl groups are close between each other and to the values of $\theta_{a}\left(\mathrm{H}_{2} \mathrm{O}\right)=$ $102^{\circ}-104^{\circ}$ on the surface of borosilicate glass plates hydrophobized in dimethyldichlorosilane vapors [53]; the surfaces of these polymers are more hydrophobic than the surfaces of fluorine-containing metathesis polynorbornenes. ULTEM 1000 and PIM-1 polyimides are also more poorly wetted by water than 6FDA fluorinated polyimide; the values of the contact angles of water drops on the surfaces of these polyimides are close between each other and to the angles on the surface of PPO. The surfaces of cellulose derivatives are the most hydrophilic; when an acetyl side group is replaced by an ethyl group, an expected increase in the maximum contact angle of water on the surface of the film is observed. It should be separately noted that the contact angles of water drops on the surfaces of the polymer films obtained by casting onto cellophane (from the side of air) and solid substrates did not differ.

A characteristic of the energy nonuniformity of the surface of a polymer film determined by the characteristic feature of its microrelief or chemical inhomogeneity is the order hysteresis of wetting; the difference of the values of the contact angles measured under the conditions of inflow and outflow $\Delta \theta=\left(\theta_{\mathrm{a}}-\theta_{\mathrm{r}}\right)$ [54]. This quantity is required to qualify the surface of the polymer and to form a sample of films with the reproducible results of the measurement of the contact angles of other liquids. The surfaces of PHFP, AF 2400, 6FDA, and PIM-1 are characterized by energy inhomogeneity among the studied polymer films.

It was assumed in some studies, the aim of which was the search for the interrelation between the surface properties and hemocompatibility of polymer materials, that the value of the maximum contact angle of water on the surface of a polymer can be considered as the simplest physicochemical parameter for the prediction of its hemocompatibility. It was empirically shown that $\theta_{\mathrm{a}}\left(\mathrm{H}_{2} \mathrm{O}\right) \approx 60^{\circ}$ is the most probable value for a hemocompatible material, which paved the way for the development of an ideology of modification of the surfaces of polymer materials by hydrophilizing reagents (phospholipids, polysaccharides, oligopeptides, substances containing poly (ethylene oxide) fragments-polyethylene glycols, Pluronics) [26, 32], and an improvement in hemocompatibility was found. When using the value of the contact angle of a water drop as the hemocompatibility prediction parameter, only ATs turns out to be a potentially hemocompatible polymer. But the presence of polar groups on the hydrophilic surface promotes good adhesion of cells [55], which does not promote hemocompatibility.

Promising hemocompatibility prediction parameters of polymer materials are the energy characteristics of the surfaces of polymer films, the specific surface free energy of the polymer/air boundary $\gamma_{\mathrm{SV}}$ and interphase energy of the polymer/liquid interface $\gamma_{\mathrm{SL}}$. 
Table 3. Energy characteristics of the surfaces of the polymer films: specific surface free energy $\gamma_{S V}$ and its dispersion $\gamma_{S V}^{d}$ and polar $\gamma_{\mathrm{SV}}^{\mathrm{p}}$ components, the interphase energy of polymer/water boundary $\gamma_{\mathrm{S}}(\mathrm{w}) \mathrm{w}$, the work of adhesion of blood cells $W_{\mathrm{a}}$, and the interphase energy of polymer/protein globule boundary $\gamma_{\mathrm{SG}}$. The dimensionally of all the quantities is [mJ $\left./ \mathrm{m}^{2}\right]$

\begin{tabular}{|c|c|c|c|c|c|c|c|c|}
\hline \multirow{2}{*}{ Polymer } & \multirow{2}{*}{$\gamma_{S V}^{\mathrm{d}}$} & \multirow{2}{*}{$\gamma_{S V}^{p}$} & \multirow{2}{*}{$\gamma_{\mathrm{SV}}$} & \multirow{2}{*}{$W_{\mathrm{a}}$} & \multicolumn{2}{|c|}{$\gamma_{\mathrm{SG}}$} & \multirow{2}{*}{$\Delta \gamma_{\mathrm{SG}}$} & \multirow{2}{*}{$\gamma_{\mathrm{S}(\mathrm{W}) \mathrm{W}}$} \\
\hline & & & & & fibrinogen & albumin & & \\
\hline PTCNSil & 38 & 0 & 38 & 76 & 42 & 34 & 8 & 23 \\
\hline PTCNSi2g & 37 & 0 & 37 & 74 & 42 & 34 & 8 & 23 \\
\hline KGO-C4F9 & 19 & 3 & 22 & 44 & 22 & 18 & 4 & 1 \\
\hline $\mathrm{KGO}-4 \mathrm{CF} 3$ & 26 & 2 & 28 & 56 & 24 & 19 & 5 & 1 \\
\hline AF 2400 & 9 & 1 & 10 & 20 & 44 & 40 & 4 & 52 \\
\hline PHFP & 14 & 0 & 14 & 28 & 42 & 37 & 5 & 51 \\
\hline PPO & 43 & 0 & 44 & 86 & 31 & 24 & 7 & 18 \\
\hline ATs & 25 & 15 & 40 & * & 6 & 4 & 2 & 6 \\
\hline ETs & 34 & 4 & 38 & 76 & 20 & 14 & 6 & 8 \\
\hline 6FDA & 40 & 6 & 46 & 92 & 17 & 12 & 5 & 2 \\
\hline ULTEM 1000 & 36 & 2 & 38 & 76 & 25 & 19 & 6 & 9 \\
\hline PIM-1 & 40 & 2 & 42 & 84 & 21 & 20 & 1 & 4 \\
\hline
\end{tabular}

* The estimate of this quantity is not correct.

These characteristics (Table 3) determine the mechanical strength of a polymer upon long-term contact with blood as well as adsorption and adhesion properties of a polymer, in this case, in relation to the components of blood [56].

For all the surfaces except for ATs, the main contribution to the value of $\gamma_{\mathrm{sv}}$ is made by the dispersion component. It should be expected that the adhesion of the blood cells on such surfaces will be executed at the expense of the comparatively weak dispersion forces; hence, it will be reversible under nonstationary conditions. The thermodynamic measure of adhesion of $W_{\mathrm{a}}=\gamma_{\mathrm{SV}}+\gamma_{\mathrm{B}}-\gamma_{\mathrm{SV}(\mathrm{B})}$, where $\gamma_{\mathrm{B}}$ is the surface energy of a biological object and $\gamma_{\mathrm{SV}(\mathrm{B})}$ is the polymer/biological object interphase energy. There are published data on the estimation of the work of adhesion of blood to various surfaces [57]. It has been shown that $W_{\mathrm{a}}$ (of blood) is significantly smaller for a hydrophobic surface (paraffin) when compared to hydrophilic surfaces (silicate glass, cellophane). It has been found that the smaller the work of adhesion of blood to the surface, the longer its coagulation time. Overall, $W_{\mathrm{a}}$ (of blood) can serve as a prediction parameter for thromboresistance; however, the data on the surface tension of blood are contradictory. Thus, according to [58], the value of the surface tension of blood $\gamma_{\mathrm{B}}=\gamma_{\mathrm{B}}^{\mathrm{d}}+\gamma_{\mathrm{B}}^{\mathrm{p}}=$ $(36.3+11.2)=47.5 \mathrm{~mJ} / \mathrm{m}^{2}$; according to the data presented in [57], $\gamma_{B}=65.1 \mathrm{~mJ} / \mathrm{m}^{2}$ and $\gamma_{\mathrm{B}}=70.5 \mathrm{~mJ} / \mathrm{m}^{2}$, and it is noted that the surface tension of blood from different donors may strongly differ. Because of this, no estimation of the value of $W_{\mathrm{a}}$ (of blood) to the surfaces of the studied polymers was performed within the scope of this study.
The data [59] on the surface tension of an erythrocyte suspension, which is $70 \mathrm{~mJ} / \mathrm{m}^{2}$ at physiological $\mathrm{pH}$ was used as the value of $\gamma_{\mathrm{B}}$ when evaluating the work of adhesion of blood cells. The estimation of $\gamma_{S V(B)}$ was performed by the Antonov rule: $\gamma_{\mathrm{SV}(\mathrm{B})}=\gamma_{\mathrm{B}}-\gamma_{\mathrm{SV}}$, which is quite correct for polymers with insignificant values of $\gamma_{\mathrm{SV}}^{\mathrm{p}}$ when the interaction between a biological object and a surface is implemented by means of dispersion forces only [56].

It should be expected based on the values of $W_{\mathrm{a}}$ that blood cells will most weakly adhere to the surfaces of PHFP, AF 2400, and KGO-C4F9.

In the study by C. Sharma [30], it was proposed to use the value of the interphase tension of polymer/protein globule boundary $\gamma_{\mathrm{SG}}$ calculated by the equations of the molecule theory of wetting using the experimental values of the surface tension of aqueous solutions of blood plasma proteins and specific surface free energy of polymers as the hemocompatibility prediction parameter. The idea of the approach consisted in the interpretation of this quantity as the energy of activation of the conformational changes of the protein globule during adsorption on a solid surface. At a minimum value of $\gamma_{\mathrm{SG}}$, the protein is not only predominantly adsorbed from the solution on the surface but also retains the native conformation in the adsorption layer. It was demonstrated that serum albumin is predominantly adsorbed on the surface of isotropic pyrolytic carbon; the material of artificial cardiac valves. Nevertheless, no clear criterion for the comparison of the values of $\gamma_{\mathrm{SG}}$ was proposed in [30] to draw a conclusion about preferable adsorption of albumin in comparison with the adsorption of fibrinogen. 
Table 4. Adsorption $\Gamma$ of plasma proteins on the surface of the polymer films and energy characteristics of the modified surfaces after rinsing in water, $[\gamma]=\mathrm{mJ} / \mathrm{m}^{2}$

\begin{tabular}{l|c|c|c|c|c|c}
\hline \multicolumn{1}{c|}{ Polymer } & PTCNSi1 & PTCNSi2g & KGO-4CF3 & PHFP & AF 2400 & PPO \\
\hline$\Gamma, \mathrm{g} / \mathrm{cm}^{2}$ & $1.7 \times 10^{-4}$ & $2.4 \times 10^{-4}$ & $1.8 \times 10^{-4}$ & $1.7 \times 10^{-4}$ & $1.6 \times 10^{-4}$ & $1.8 \times 10^{-4}$ \\
$\Gamma, \mathrm{wt} \% *$ & 90 & 93 & 78 & 79 & 72 & 88 \\
$\gamma_{\mathrm{SV}}^{\mathrm{d}}$ & 38 & 36 & 36 & 33 & 35 & 38 \\
$\gamma_{\mathrm{SV}}^{\mathrm{p}}$ & 1 & 2 & 2 & 6 & 10 & 1 \\
$\gamma_{\mathrm{SV}}$ & 39 & 38 & 38 & 39 & 45 & 39 \\
\hline
\end{tabular}

* The degree of retention of the adsorption layer on the surface of the polymer after rinsing.

The greatest difference between the values of $\gamma_{\mathrm{SG}}$ for fibrinogen and albumin is observed for polymers of the PTNCSi and PPO series (Table 3). In accordance with the approach [30], it can be expected that albumin will predominantly adsorb on the surface of these polymers, and its adsorption layer will inhibit the adhesion of platelets. However, these polymers are characterized by practically the highest out of all estimated values of $W_{\mathrm{a}}$ of blood cells. PHFP, KGO-4CF3, ULTEM, PIM-1, and ETs are the polymers demonstrating medium indices with respect to the difference of the values of $\gamma_{\mathrm{SG}}$ but, at the same time, characterized by insignificant $W_{\mathrm{a}}$ (Table 3 ).

The main idea of the approach of E. Ruckenstein [29], according to which a polymer with the criterial value of the interphase energy at the boundary with water $\gamma_{\mathrm{S}(\mathrm{W}) \mathrm{W}}=1-3 \mathrm{~mJ} / \mathrm{m}^{2}$ is potentially hemocompatible, is the creation of a thermodynamic prohibition for the adsorption of any plasma protein on the surface in contact with blood. In this case, the interphase energy of the polymer/water surface should be minimum but, here, the thermodynamic conditions for the spontaneous dispersion of the polymer into the liquid medium should not be provided. In accordance with the approach and procedure of E. Ruckenstein, good hemocompatibility parameters are demonstrated by the polymers of the KGO, 6FDA, and PIM-1 series (Table 3).

It has been shown by PW that proteins are well adsorbed from blood plasma on the surface of the polymers irrespective of the value of $\gamma_{\mathrm{s}(\mathrm{W}) \mathrm{W}}$ (Table 4). The values of adsorption on the surface of the polymer films are close between each other except for PTCNSi2g for which the adsorption is the highest. The specific surface free energy of all the studied films modified by the adsorption layers of plasma proteins is $\gamma_{\mathrm{SV}}=\gamma_{\mathrm{SV}}^{\mathrm{d}}+$ $\gamma_{\mathrm{SV}}^{\mathrm{p}}=(37+1)=38 \mathrm{~mJ} / \mathrm{m}^{2}$. Intensive rinsing of the polymer films in water for 5 min (the imitation of a flux) leads to the removal of the proteins adsorbed from plasma approximately from 10 to $30 \mathrm{wt} \%$. The adsorption layers of plasma proteins are to the greatest extent retained on the surface of the polymers of the PPO and PTCNSi series; the layers are the most effectively removed from the surfaces of fluorinated polymers, namely, KGO-4CF3, PHFP, and AF 2400.

The energy characteristics of the surfaces of the polymers modified by the adsorption layers of plasma proteins noticeably change after rinsing only for PHFP and AF 2400. This result indirectly indicates the difference in the composition of the adsorption layers of plasma proteins on the surface of these polymers from the layers of the surfaces of $\mathrm{KGO}-4 \mathrm{CF} 3$ and polymers of the PTCNSi and PPO series. The increase in $\gamma_{\mathrm{SV}}^{\mathrm{p}}$, especially in the case of AF 2400, can promote the intensification of the adhesion of blood cells due to their polar interactions with the modified surface.

The analysis for free hemoglobin shows the degree of traumability of erythrocytes upon contact with a sample of a polymer (Table 5), thus, the higher the index in comparison to the control, the higher the traumability of erythrocytes. In the experiment, the gain in free hemoglobin was determined by the difference of the concentration of free hemoglobin $\left(C_{\mathrm{Hb}}, \mathrm{g} / \mathrm{L}\right)$ with the control $\left(\Delta C_{\mathrm{Hb}}, \mathrm{g} / \mathrm{L}\right)$. Since the values of $C_{\mathrm{Hb}}$ in the control samples substantially differed in different experiments, the relative gain in free hemoglobin normalized to the value of $C_{\mathrm{Hb}}$ in the control samples $\left(\Delta C_{\mathrm{Hb}}, \%\right.$ of the control) was used for comparing the experimental data. The rank was determined in accordance with the "place" occupied in the sample with respect to the value of $\Delta C_{\mathrm{Hb}}, \%$. Table 5 presents the results of expert evaluation: erythrocytes are not traumatized ("Excellent"), erythrocytes are insignificantly traumatized ("Good"), erythrocytes are to a certain extent traumatized ("Satisfactory"), erythrocytes are significantly traumatized ("Unsatisfactory").

According to the obtained data, PHFP, KGO-C4F9, and PTNCSil possess the best hemocompatibility. Acceptable hemocompatibility is demonstrated by PPO and PIM-1. KGO-4CF3, PTNCSi2g, and ETs fall into the group of polymers that lead to certain traumability of erythrocytes. Eventually, ULTEM 1000 and 6FDA polyimides as well as ATs and AF 2400 turn out to be unacceptable for application based on the data of the analysis for free hemoglobin. 
Table 5. Results of the analysis for free hemoglobin

\begin{tabular}{|c|c|c|c|c|}
\hline Polymer & $\Delta C_{\mathrm{Hb}}, \mathrm{g} / \mathrm{L}$ & $\Delta C_{\mathrm{Hb}}, \%$ of the control & Rank by $\%$ & Expert evaluation \\
\hline PTNCSil & 0.0112 & 4.7 & 3 & "Excellent" \\
\hline PTNCSi2g & 0.0756 & 32.0 & 7 & "Satisfactory" \\
\hline KGO-C4F9 & -0.0086 & -3.6 & 2 & "Excellent" \\
\hline KGO-4CF3 & 0.0751 & 31.8 & 6 & "Satisfactory" \\
\hline AF 2400 & 0.1727 & 73.0 & 11 & "Unsatisfactory" \\
\hline PHFP & -0.00585 & -5.6 & 1 & "Excellent" \\
\hline PPO & 0.01195 & 11.5 & 4 & "Good" \\
\hline ATs & 0.10635 & 102.5 & 12 & "Unsatisfactory" \\
\hline ETs & 0.04165 & 40.1 & 8 & "Satisfactory" \\
\hline $6 \mathrm{FDA}$ & 0.06535 & 63.0 & 9 & "Unsatisfactory" \\
\hline ULTEM 1000 & 0.06835 & 65.9 & 10 & "Unsatisfactory" \\
\hline PIM-1 & 0.0294 & 12.4 & 5 & "Good" \\
\hline
\end{tabular}

\section{The Morphofunctional Study of Blood Cells}

The study of erythrocytes. Upon contact of blood with the samples under study, no pronounced destruction of erythrocytes was observed in all the cases, and the fraction of degenerate cells among all the erythrocytes did not exceed 5\%. In the presence of the samples of PPO, PIM-1, PTNCSi2g, PTNCSi1, KGO-4CF3, and PHFP, the ratio of the main morphological types of erythrocytes did not significantly differ from the control (Fig. 1a). A reliable increase in the fraction of cells with uneven edges and echinocytes was noted in the experiments with the samples of ULTEM 1000,
AF 2400, and 6FDA (Fig. 1b). Here, the fraction of normal erythrocytes decreased 1.12-fold on average, i.e., no sharp change in the structure of the erythrocyte population occurred. Also, an insignificant decrease in the fraction of normal erythrocytes was noted in the experiments with the samples of KGO-C4F9, ATs, and ETs. Overall, it can be concluded that the presented materials are low-toxic for human erythrocytes under the conditions of exposure in vitro.

The study of platelets. The morphofunctional status of platelets did not undergo visible changes in the experiments with the samples of PPO, KGO-4CF3,
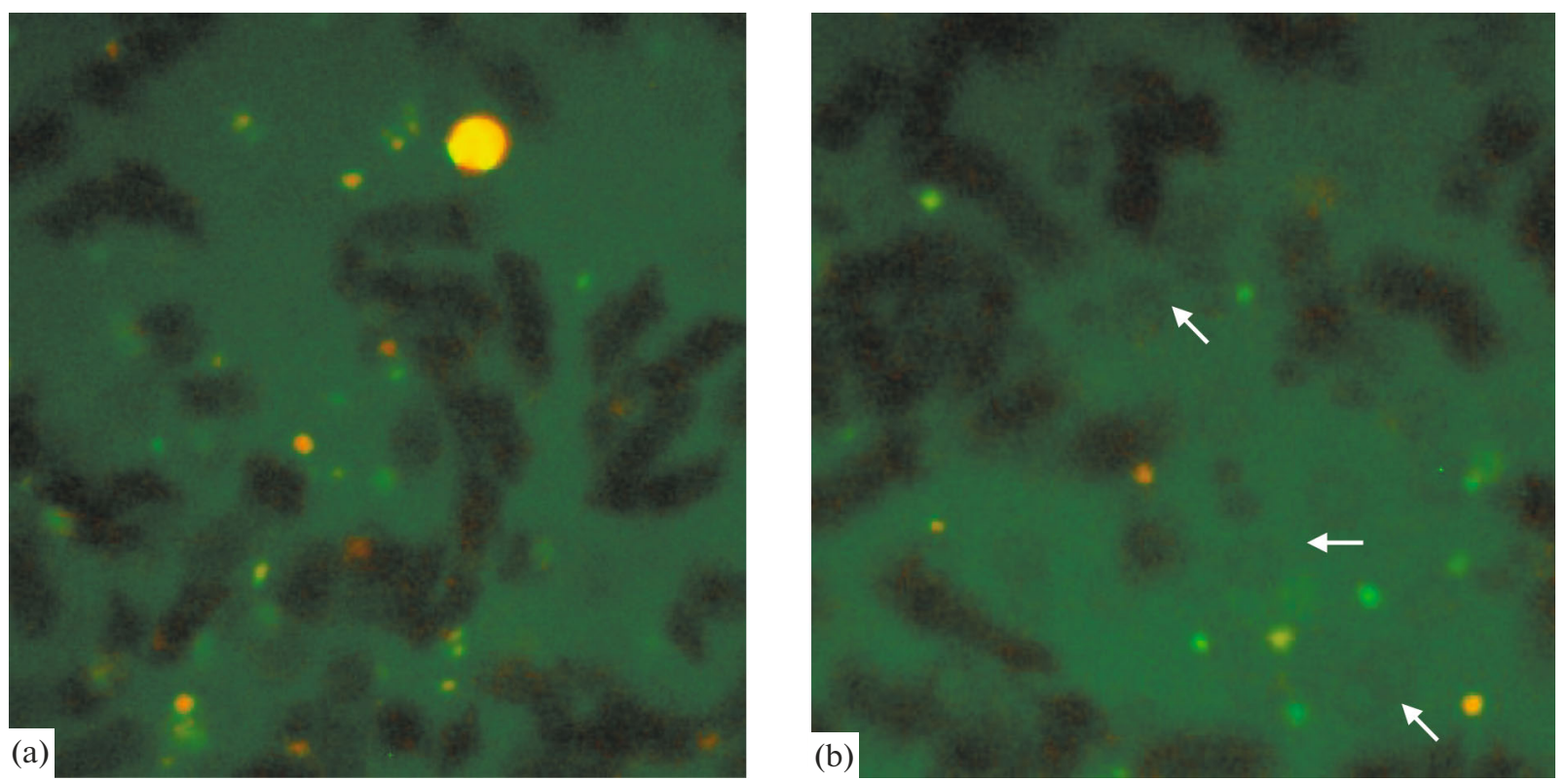

Fig. 1. Vital staining of blood cells after contact with polymer films. The magnification is $\times 800$. Staining by trypaflavine-acridine orange. (a) PPO-the platelets and leucocytes have normal morphology; the erythrocytes are not stained. (b) AF $2400-$ the arrows indicate the erythrocytes with damaged membranes which diffusely distribute the stain throughout the entire volume; the number of platelets with granules simultaneously decreases. 


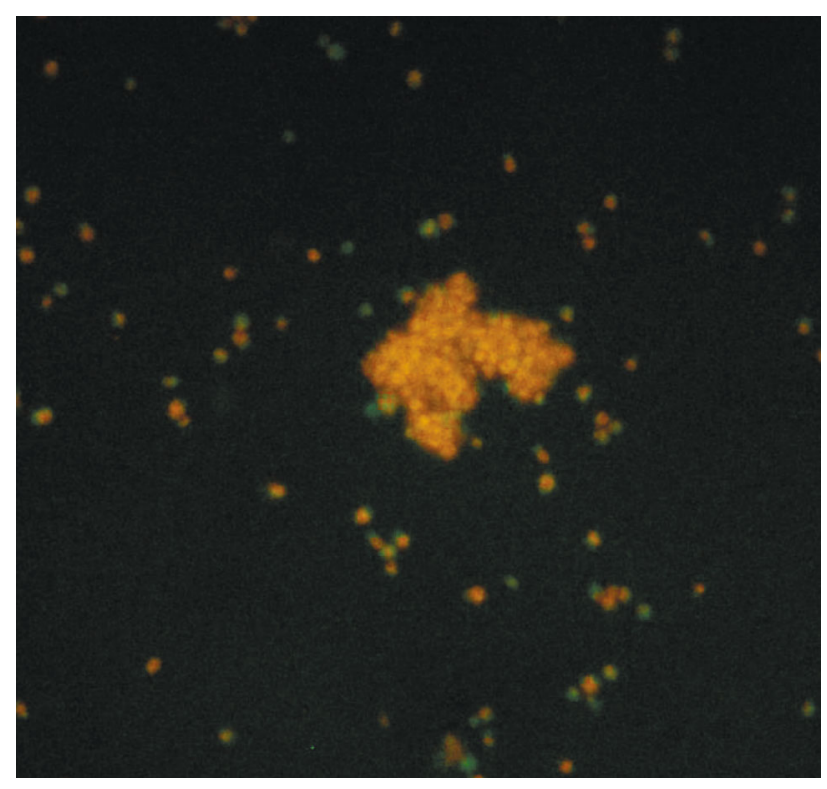

Fig. 2. Vital staining of blood cells after a contact with a PIM-1 film. The magnification is $\times 400$. Staining by trypaflavine-acridine orange. Spontaneous formation of platelet aggregates and degranulation of platelets are observed in blood.

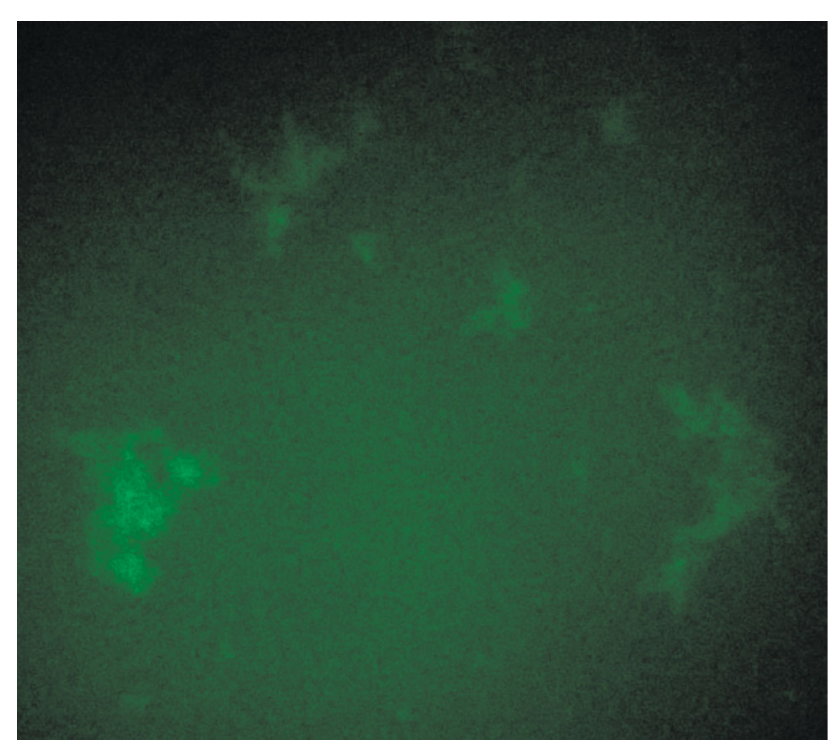

Fig. 3. Detection of platelet aggregates on the surface of AF 2400 films. The magnification is $\times 100$. Staining by trypaflavine-acridine orange.

PTNCSi1, and PHFP. After contact of platelets with these polymers, biologically native platelets retained their structural integrity, and no release of platelet granules occurred. An insignificant decrease (by 2$6 \%$ ) in the fraction of platelets with granules without visible violation of the structural integrity of their membranes was noted in the experiments with the samples of ULTEM 1000, 6FDA, PTNCSi2g, ATs, and ETs. On the opposite, in the experiments with the AF 2400, PIM-1, and KGO-C4F9 materials, the qualities of platelets noticeably decreased, thus, the fraction of the cells with granules decreased 1.5-3-fold in comparison with the control, and the formation of platelet aggregates in blood was simultaneously observed (Fig. 2). In all the experiments with the PIM-1 and AF 2400 materials, adhered platelet aggregates with a diameter of up to $50 \mu \mathrm{m}$ could be seen on the surface of the sample under study (Fig. 3). There were no platelet aggregates on the rest of the materials.

It can be concluded that the films of PPO, KGO-4CF3, PTNCSi1, and PHFP possess pronounced thromboresistance and do not induce spontaneous activation of platelets in blood in vitro. The films of ULTEM 1000, 6FDA, PTNCSi2g, ATs, and ETs induce an insignificant decrease in the quality of platelets without their spontaneous activation, while pronounced aggregation of platelets in the suspension and on the surface of the films is observed in the experiments with PIM-1 and AF 2400. In the experiments with KGO-C4F9, spontaneous activation of platelets was not as pronounced; however, like in the case of PIM-1 and AF 2400, degranulation of many platelets was observed. The release of granules by platelets means their transition to the irreversibly activated state, which may increase the risk of development of thrombotic complications in vivo. Therefore, the AF 2400, PIM-1, and KGO-4CF3 materials create a potential risk of spontaneous activation of platelets and are not thromboresistant.

The study of leucocytes. In the experiments with the samples of AF 2400, PHFP, PTNCSi2g, PTNCSi1, $\mathrm{KGO}-4 \mathrm{CF} 3$, and KGO-C4F9, the fraction of damaged leucocytes was the same as in the control, and in the experiments with the samples of ULTEM 1000, 6FDA, ATs, and ETs, the fraction of damaged leucocytes was higher than the initial fraction by just $1-2 \%$. No decrease in the brightness of leucocytes, their degranulation, or formation of leucocyte-platelet conglomerates was observed in any of the experiments. Therefore, all the proposed materials possess low toxicity for human leucocytes under conditions of exposure in vitro.

The results of the morphofunctional analysis of the state of blood cells are summarized in Table 6 .

The estimates of the direct methods of determination of hemocompatibility insignificantly diverge. Based on the cumulative estimates of these methods, only two polymers, namely, PHFP and PTNCSi1, should be recognized as hemocompatible polymers. PPO and, possibly, KGO-4CF3 also fall within the group of highly promising polymers. All methods clearly indicate that AF 2400 is unsuitable for use as the material for ECMO. 
Table 6. Results of the evaluation of the biocompatibility of the polymers with blood cells by biomedical methods

\begin{tabular}{l|l}
\hline $\begin{array}{l}\text { Highly compatible (do not induce any visible change in the blood } \\
\text { cells in vitro, are thromboresistant, are the most preferable } \\
\text { for use as the material for the oxygenator membrane) }\end{array}$ & PPO, PTNCSi1, KGO-4CF3, and PHFP \\
$\begin{array}{l}\text { Medium-compatible (do not induce any sharp change } \\
\text { in the blood cells in vitro, do not induce spontaneous } \\
\text { aggregation of platelets) }\end{array}$ & PTCNSi2g, ULTEM 1000, ATs, ETs, and 6FDA \\
$\begin{array}{l}\text { Low-compatible (sharply change the properties of the cells } \\
\text { in vitro, do not possess thromboresistance) }\end{array}$ & AF 2400, PIM-1, and KGO-C4F9
\end{tabular}

\section{CONCLUSIONS}

The analysis of the values of the maximum contact angles of water (Table 2) on the surfaces of the polymer films and data of biomedical studies shows that $\theta_{\mathrm{a}}\left(\mathrm{H}_{2} \mathrm{O}\right) \approx 60^{\circ}$ cannot be a parameter for the evaluation of the hemocompatibility of a material even in the first approximation; polytricyclononenes and fluorine-containing hydrophobic polymers as well as semicrystalline PPO possess the best indices for the provision of hemocompatibility based on the biomedical tests (Table 1). Here, the experimental fact that surfaces similar in the chemical composition and water wettability (PHFP, AF 2400, polytricyclononenes, polynorbornenes of the KGO series) demonstrate different hemocompatibilities up to principal differences (PHFP, AF 2400) remains completely unclear.

The comparison of the energy characteristics of the surfaces of the polymer films (Table 4) with the results of morphofunctional analysis and adhesion of blood cells (Table 6) indicates that the lowest adhesion and, as a consequence, the smallest morphological changes in the studied objects are observed for the polymer films with the smallest polar component of the specific surface free energy. This means that conditions for the implementation of the interactions of blood cells with the surface solely due to the dispersion forces should be provided when creating a hemocompatible material. This means that the surface of the polymer should be limitedly wetted by water. On the other hand, the value of the interphase energy at the boundary of such a polymer with an aqueous medium is the moving force of adsorption of plasma proteins on the surface of the polymer. When considering this factor, it is reasonable to use the approach of C. Sharma and experimentally confirm it. One should be careful to use the Ruckenstein criterion when predicting the hemocompatibility of polymer materials because this approach gives contradictory results [60]. Hemolysis, i.e., formation of free hemoglobin, also results from the adhesion of erythrocytes. However, it has turned out to be impossible to find any clear correlation between the values of $W_{\mathrm{a}}$ (Table 4 ) and analysis for free hemoglobin (Table 5) for the studied polymers, which may be associated with a more complex mechanism of traumability of erythrocytes in comparison with the change in the morphology of blood cells.
The complexity of the mechanism of interaction of the surface of polymers with blood does not allow for clear structure-property correlations traditional for membrane gas separation $[61,62]$ despite the fact that groups of polymers with related chemical structures of the elementary units were used in the study. Thus, for additive polytricyclononenes containing trimethylsilyl groups, less permeable (Table 1) PTNCSi1 turns out to be a polymer more promising for ECMO, while more permeable PTNCSi2g does not fall within the sample of hemocompatible polymers. At the same time, the highest adsorption of plasma blood proteins is characteristic for PTNCSi2g (Table 4). Apparently, the microporous structure of this polymer [14, 41] leads to an increased sorption of low-molecularweight blood components and, subsequently, the violation of the sorption-desorption processes. Possibly, because of this, there is an upper limit of gas permeability for glass-like polymers, starting from which a polymer becomes unsuitable for application as a diffusion coating. This problem can be solved by additional studies of the polymers with $P\left(\mathrm{O}_{2}\right)>1000$ Barrer, i.e., of the series of polynorbornenes and polyacetyelenes $[13,14]$. Once again, in the group of metathesis fluorine-containing polynorbornenes, KGO-4CF3 fell within the group of relatively promising polymers, while KGO-C4F9 turned out to be low compatible. Possibly, the reason consists in the low glass transition point of KGO-C4F9. Nevertheless, additional studies for this promising group of polymers should also be continued. The situation in the group of perfluorinated polymers is also unclear, thus, PHFP is the most hemocompatible out of the studied polymers, which confirms the conclusions of [17]; however, more permeable AF 2400 turned out to be the worst of the studied polymers with respect to all the indices, although the surface characteristics of these polymers are almost indistinguishable. An indirect answer to this question is the sharp increase in the polarity of the surface of AF 2400 modified by blood plasma proteins (Table 4). The studies in this direction for the explanation of the found contradictions should also be continued. In the groups of semicrystalline polymers, cellulose esters expectedly turned out to be low-compatible with blood. However, semicrystalline ester PPO fell within the group of polymers promising for ECMO. Possibly, in this case, the specific structure of the low- 
density crystalline phase plays a special role like for PMP, which also requires additional studies. At the same time, an unambiguous conclusion can be made for polyimides containing nitrogen and oxygen heteroatoms that this group of polymers is unsuitable for ECMO irrespective of the level of permeability. A similar conclusion can also be made for polymers with internal microporosity. For these polymers that also contain nitrogen and oxygen heteroatoms, increased sorption of the low-molecular-weight blood components and violations of the sorption-desorption processes are also possible like in the case of PTNCSi2g due to the microporous structure of the polymer.

\section{FUNDING}

This study was performed as part of a State Task to the Topchiev Institute of Petrochemical Synthesis, Russian Academy of Sciences and within state-funded topic no. AAAA-A16-116030250108-3 in part of determination of the energy characteristics of the surfaces of polymer films.

\section{REFERENCES}

1. S. V. Got'e, V. N. Poptsov, and E. A. Spirina, Extracorporeal Membrane Oxygenation in Cardiac Surgery and Transplantology (Triada, Moscow, Tver, 2013) [in Russian].

2. A. K. Evseev, S. V. Zhuravel, A. Yu. Alentiev, I. V. Goroncharovskaya, and S. S. Petrikov, Memb. Membr. Technol. 1, 201 (2019).

3. S. V. Zhuravel', D. A. Kosolapov, and M. V. Ketskalo, Transplantologiya, 4, 28 (2014).

4. http://экмо.pф/en/.

5. www.euroelso.net/covid-19/covid-19-survey/

6. www.euroelso.net/inhalt/uploads/2020/06/Map-202006-21.pdf

7. W. L. Robb, Ann. NY Acad. Sci, 146, 119 (1968).

8. V. V. Zhmakin and V. V. Teplyakov, Sep. Purif. Technol. 186, 145 (2017).

9. E. Khoshbin, C. Westrope, S. Pooboni, D. Machin, H. Killer, G. J. Peek, A. W. Sosnowski, and R. K. Firmin, Perfusion 20, 129 (2005).

10. M. C. Bélanger and Y. Marois, J. Biomed. Mater. Res. 58, 467 (2001).

11. L.W. McKeen, Permeability Properties of Plastics and Elastomers (4th Ed., Elsevier, Amsterdam, 2017).

12. Gas Separation Parameters of Glassy Polymers, Database (Informregistr RF, 1998, no. 3585).

13. T. Sakaguchi and T. Masuda, in Membrane Materials for Gas and Vapor Separation. Synthesis and Application of Silicon-Containing Polymers (Yampolskii Yu., Finkelshtein E., Eds., Chichester, Wiley, 2017).

14. E. Finkelshtein, M. Gringolts, M. Bermeshev, P. Chapala, and Yu. Rogan, in Membrane Materials for Gas and Vapor Separation. Synthesis and Application of SiliconContaining Polymers (Yampolskii Yu., Finkelshtein E., Eds., Chichester, Wiley, 2017).

15. T. C. Merkel, I. Pinnau, R. Prabhakar, and B. D. Freeman, in Materials Science of Membranes for Gas and Vapor
Separation (Yampolksi Yu., Pinnau I., Freeman B. D., Eds., Wiley, Chichester, 2006).

16. Z.-X. Low, P. M. Budd, N. B. McKeown, and D. A. Patterson, Chem. Rev. 118, 5871 (2018).

17. A. Yu. Alentiev, V. E. Ryzhikh, and N. A. Belov, Polym. Sci., Ser. C (2020) (in press).

18. A. Yu. Alentiev, N. A. Belov, R. Yu. Nikiforov, E. V. Polunin, N. V. Borovkova, A. K. Evseev, M. S. Makarov, I. V. Goroncharovskaya, M. V. Storozheva, and V. S. Zhuravel, Petr. Chem. 58, 740 (2018).

19. Hemocompatibility of Biomaterials for Clinical Applications: Blood-Biomaterials Interactions, Ed. by Siedlecki C. (Cambridge, Woodhead Publishing, 2018).

20. F. Obstals, M. Vorobii, T. Riedel, A. d. L. S. Pereira, M. Michael Bruns, S. Singh, and C. Rodriguez-Emmenegger, Macromol. Biosci. 18, 3 (2018).

21. A. J. Doyle and B. J. Hunt, Front. Med. 5, 352 (2018).

22. M. Weber, H. Steinle, S. Golombek, L. Hann, C. Schlensak, H. P. Wendel, and M. Avci-Adali, Front. Bioeng. Biotech. 6, 99 (2018).

23. G. Altankov and T. Groth, J. Mater. Sci. Mater. Med. 7, 425 (1996).

24. C. Zhao, X. Liu, M. Nomizu, and N. Nisho, Biomaterials 24, 3747 (2003).

25. Yu. Wu, F. I. Simonovsky, B. D. Ratner, and T. A. Horbett, J. Biomed. Mater. Res. 74A, 722 (2005).

26. G. P. Yampol'skaya and V. D. Dolzhikova, Vestn. Mosk. Univ., Ser. 2: Khim. 48, 33 (2007).

27. S. L. West, J. P. Salvage, E. J. Lobb, S. P. Armes, et al., Biomaterials 25, 1195 (2004).

28. E. D. Shchukin, A. V. Pertsov, and E. A. Amelina, Colloidal Chemistry (Moscow: Vysshaya shkola, 2004) [in Russian].

29. E. Ruckenstein and S. V. Gourisankar, Biomaterials 17, 403 (1986).

30. L. Paul and C. P. Sharma, J. Colloid Interface Sci. 84, 456 (1981).

31. J. Kloubek, Adv. Colloid Interface Sci. 38, 99 (1992).

32. Yu. G. Bogdanova and V. D. Dolzhikova, in Proceedings of the XVI All-Russian Conference "Structure and dynamics of molecular systems, " V. 3, MarGTU, IoshkarOla, 2009, p. 4.

33. N. B. Tavakalyan, A. G. Karapetyan, A. S. Pogosyan, A. K. Abrahamyan, A. Corti, A. Pompella, and A. Mihranyan, J. Mater. Sci.: Mater. Med. 21, 1693 (2010).

34. A. Marmur, J. Colloid Interface Sci. 97, 585 (1984).

35. Y. C. Nho and O. H. Kwon, Radiat. Phys. Chem. 66, 299 (2003).

36. N. M. Tran, T. L. Huynh, B. N. Phan, N. N. Dang, T. B. Phan, H. Ha, L. P. Truong, P. D. Huynh, N. Q. Tran, V. T. Vo, and P. T.-H. Nguyen, Int. J. Polymer Sci. 1 (2020).

37. S. R. Schricker, M. Palacio, and B. Bhushan, Modulating Protein Adhesion and Conformation with Block Copolymer Surfaces. Handbook of Nanomaterials Properties (2014).

38. K. Vijayanana, D. K. Pattanayak, T. R. Mohan Rama, and R. Banerjee, Trends Biomater. Artif. Organs 18, 73 (2005). 
39. I. Stoica, A. I. Barzic, and C. Hulubei, in 5th IEEE International Conference on E-Health and Bioengineering EHB, Romania (2015).

40. M. Gringolts, M. Bermeshev, Yu. Yampolskii, L. Starannikova, V. Shantarovich, and E. Finkelshtein, Macromolecules 43, 7165 (2010).

41. P. P. Chapala, M. V. Bermeshev, L. E. Starannikova, N. A. Belov, V. E. Ryzhikh, V. P. Shantarovich, V. G. Lakhtin, N. N. Gavrilova, Yu. P. Yampolskii, and E. Sh. Finkelshtein, Macromolecules 48, 8055 (2015).

42. G. O. Karpov, M. V. Bermeshev, I. L. Borisov, S. R. Sterlin, A. A. Tyutyunov, N. P. Yevlampieva, B. A. Bulgakov, V. V. Volkov, and E. Sh. Finkelshtein, Polymer 153, 626 (2018).

43. S. M. Nemser and I. A. Roman, US Patent 5051114 (1991).

44. A. Yu. Alentiev, Yu. P. Yampolskii, V. P. Shantarovich, S. M. Nemser, and N. A. Plate, J. Membr. Sci. 126, 123 (1997).

45. N. A. Belov, A. A. Zharov, A. V. Shashkin, M. Q. Shaikh, K. Raetzke, and Yu. P. Yampolskii, J. Membr. Sci. 383, 70 (2011).

46. A. A. Kuznetsov, A. Yu. Tsegelskaya, A. M. Orlova, N. A. Belov, S. V. Chirkov, R. Yu. Nikiforov, and A. Yu. Alentiev, Membr. Membr. Technol. 1, 316 (2019).

47. A. Yu. Alentiev, N. A. Belov, S. V. Chirkov, and Yu. P. Yampolskii, J. Membr. Sci. 547, 99 (2018).

48. I. I. Ponomarev, D. Yu. Razorenov, I. V. Blagodatskikh, A. V. Muranov, L. E. Starannikova, A. Yu. Alent'ev, R. Yu. Nikiforov, and Yu. P. Yampol'skii, Polym. Sci., Ser. B 61, 369 (2019).
49. G. Sauerbrey, Z. Physyk 155, 206 (1959).

50. M. Harboe, Scand. J. Clin. Lab. Invest. 11, 66 (1959).

51. M. S. Makarov, V. B. Khvatov, O. I. Konyushko, N. V. Borovkova, M. V. Storozheva, and I. N. Ponomarev, RF Patent No. 2484472, Byull. No. 16 (2013).

52. M. Sh. Khubutiya, M. S. Makarov, V. B. Khvatov, I. V. Vysochin, E. N. Kobzeva, N. V. Borovkova, and O. I. Konyushko, RF Patent No. 2485502, Byull. No. 17 (2013).

53. Yu. G. Bogdanova, V. D. Dolzhikova, and B. D. Summ, Vestn. Mosk. Univ., Ser. 2: Khim. 45, 186 (2004).

54. P. Zh. De Zhen, Usp. Fiz. Nauk 151, 619 (1987).

55. J. L. Dewez, A. Doren, Y. J. Schneider, and P. G. Rouxhet, Biomaterials 20, 547 (1999).

56. A. Adamson, Physical Chemistry of Surfaces (New York: Wiley, 1976).

57. A.D. Zimon, Adhesion of Liquids and Wetting (Moscow: Khimiya, 1974).

58. S. Agathopoulos and P. Nikolopoulos, J. Biomed. Mater. Res 29, 421 (1995).

59. P. V. Mokrushnikov, Byull. SB RAMS 30, 53 (2010).

60. Yu. G. Bogdanova, V. D. Dolzhikova, and A. G. Mazhuga, Klei, Germetiki, Tekhnol., 7, 2 (2010).

61. A. Alentiev, Yu. Yampolskii, V. Ryzhikh, and D. Tsarev, Membr. Membr. Technol. 53, 554 (2013).

62. V. Ryzhikh, D. Tsarev, A. Alentiev, and Yu. Yampolskii, J. Membr. Sci. 487, 189 (2015).

Translated by E. Boltukhina 